INVESTIGACIONES

\title{
¿QUE HACE A LA FORMACION PERMANENTE DE PROFESORES EFICAZ?: FACTORES QUE INCIDEN EN SU IMPACTO ${ }^{1}$
}

\author{
WHAT MAKES CONTINUOUS TEACHER EDUCATION EFFICIENT?: \\ FACTORS THAT ASSFFECT ITS INFLUENCE \\ O que faz a formação permanente dos professores ser eficaz?: \\ Fatores que incidem no seu impacto
}

\author{
Christian Miranda J.*, Pablo Rivera R., Silvia Salinas, Erwin Muñoz B. \\ *christianmiranda@uach.cl
}

\begin{abstract}
RESUMEN
Este artículo examina la incidencia de las características estructurales y de proceso en el impacto de los Programas de Postítulo (PP) sobre el conocimiento, práctica, aprendizaje estudiantil y eficacia según el reporte de los docentes beneficiarios de la cohorte 2007.

Metodológicamente el estudio incluye 568 docentes que participaron en módulos presenciales (600 horas) y seminarios grupales (275 horas), donde los profesores actualizan conocimientos disciplinario-pedagógicos y diseñan propuestas de intervención educativas, mientras desempeñan en paralelo labores de aula.

Los resultados sugieren que los PP cumplen su propósito de optimizar el Conocimiento del contenido y que profesores pares y líderes de los colegios de procedencia tendrían una influencia penetrante y decisiva en los factores que inciden en la confianza, puesta en práctica y posible transferencia que los docentes beneficiarios tienen para satisfacer las necesidades de aprendizaje de sus estudiantes y, por ende, en el mejoramiento de la calidad educativa.
\end{abstract}

Palabras clave: Formación permanente de profesores, eficacia, impacto.

\begin{abstract}
This article examines the incidence of the structural and process related characteristics of the impact of Postgraduate Programs (PP) on the knowledge, practice, student learning and effectiveness, according to the report obtaineds from the teachers belonging to the cohort 2007. Methodologically speaking, the study includes 568 teachers who attended classes (600 hours) and group seminars $(275 \mathrm{hrs})$, in which the teachers updated their subject-pedagogical knowledge and designed teaching intervention projects, while they continued working at their schools.

The results suggest that Postgraduate courses fulfil their aim to optimize content knowledge. In addition, results also showed that teacher peers and leaders from the involved schools had an incisive and crucial influence on factors that affect self confidence, teaching practice, and possible transference into the classroom to satisfy students' learning needs and, therefore, improving the quality of education.
\end{abstract}

Key words: Continuous teacher education, effectiveness, impact.

\section{RESUMO}

Este artigo examina a incidência das características estruturais e de processo no impacto dos Programas de Póstítulo (PP) sobre o conhecimento, a prática, a aprendizagem estudantil, e a eficácia conforme o informe dos professores beneficiados da coorte 2007.

1 Este artículo forma parte de la investigación FONDECYT N ${ }^{\circ} 11060128$, financiado por CONICYT. 
Metodològicamente, o estudo inclui 568 professores que participaram em módulos presenciais (600 horas) e em seminários grupais (275 horas), nos que os professores atualizaram conhecimentos de disciplina pedagógica e desenharam propostas de intervenção educativas, em quanto seguiam dando aulas simultaneamente.

Os resultados sugerem que os PP cumprem seu propósito de aperfeiçoar o Conhecimento do conteúdo e que os professores pares e os líderes dos colégios de procedência teriam uma influencia penetrante e decisiva nos fatores que incidem na confiança, na posta em prática e possível transferência que os professores beneficiados têm para satisfazer as necessidades de aprendizagem dos seus estudantes e, por tanto, no melhoramento da qualidade educativa.

Palavras-chave: Formação permanente de professores, eficácia, impacto.

\section{INTRODUCCION}

Una de las metas actuales de Chile es acceder al grupo de los países desarrollados. En efecto, en el año 2000 las autoridades expresaban que para el 2010 el país tendría un desarrollo similar al de España. Luego, transcurrido más de un lustro de aquel augurio, la meta no sólo se ha moderado (el estándar es Portugal) sino que se ha aplazado para el año 2020. Ante este nuevo escenario, la pregunta evidente es ¿qué factores inciden en el cambio en la meta de desarrollo?

Una respuesta a esta interrogante es que al país le falta "competitividad". Si bien mantiene su liderazgo competitivo en América Latina, está lejos de las principales economías mundiales (Núñez, 2008). El concepto de "competitividad" recientemente se ha vuelto clave, pues se relaciona con la "innovación" entendida como hacer las cosas de una manera distinta que el resto. Sobre este punto, Martínez (2008) consultó a cien gestores de los distintos sectores productivos, académicos y profesionales del país sobre los factores que obstaculizan la competitividad y las tareas pendientes. El 51\% de las personas consultadas marcó a la educación como la primera amenaza a la competitividad y el $93 \%$ indicó que la educación es un área que se debe potenciar si queremos ser un país desarrollado para el año 2020. ¿Por qué la educación es citada como tan importante en el desarrollo de la innovación en el país?

Esto se debe a que la educación tiene como función formar ciudadanos competentes en las dimensiones del saber que conforman, por ejemplo, la ciencia y la tecnología que permitirán sustentar el vertiginoso avance de la sociedad actual. Estas dimensiones del saber corresponden a los conocimientos, habilidades y actitudes (Pozo, 2006) que el y la estudiante deben desarrollar con ayuda de sus profesores. Sin embargo, y como se muestra en estudios nacionales (Castillo y Cerda, 2006) e internacionales (OCDE, 2004 y 2009), diferentes indicadores arrojan que los niños y niñas de nuestro país no están teniendo el éxito esperado y que tampoco lo están haciendo los profesores encargados de formarlos.

Sobre estos últimos, se constata una brecha de capacidad entre los requerimientos del currículum escolar y su formación de base. Especial referencia se hace a los docentes que laboran en los últimos niveles de la educación primaria, donde áreas del currículum de alto impacto como las matemáticas y las ciencias son enseñadas por docentes cuya formación generalista no los prepara adecuadamente para su conocimiento disciplinar ni la adecuación didáctica necesaria para hacer enseñable estos conocimientos asociados a la alfabetización científica. Sobre ello, una línea de investigación se ha cuestionado sobre esta brecha de capacidad, desde la mirada a esfuerzos sistemáticos por generar acciones compensatorias a este problema, tales como el Programa de Postítulo de mención 
direccionado por el CPEIP con apoyo de universidades formadoras de profesores. Al respecto la investigación (Miranda et al., 2008) es consistente en señalar la efectividad de estos programas en relación a las oportunidades de aprendizaje disciplinar que desarrollan, pero sostienen la necesidad de preguntarse por qué factores inciden en esta efectividad. Este es el objeto de la presente investigación.

A continuación se presenta la base conceptual, desarrollo metodológico y principales resultados del estudio, a fin de tensionar y discutir el rol que los factores de procedencia, las características estructurales y oportunidades para aprender de los programas y la comunidad profesional tienen al momento de condicionar o favorecer el éxito o fracaso de tales propuestas de formación permanente de profesores.

\section{ANTECEDENTES CONCEPTUALES}

La evaluación de programas de formación permanente de docentes presenta retos aún no resueltos: como por ejemplo, develar el origen teórico de las propuestas y evaluar su eficacia. ¿Qué y cuándo se evalúa y quién debe hacerlo? Estas son preguntas propias de todos los procesos de evaluación, pero cuando se trata de los programas de formación permanente de profesores, aparecen las primeras reacciones en contra de inversiones que son muy costosas y con pocos resultados (OCDE, 2004). Es aquí donde los indicadores se multiplican, se hace más difícil verificar su cumplimiento y el impacto parece diluirse. Es clave, entonces, formularse las interrogantes adecuadas teniendo en cuenta los contextos de desarrollo de los programas.

\section{I.1. EVALUACION: CONCEPTO Y ENFOQUES EN USO SOBRE LA FORMACION PERMANENTE}

Desde el punto de vista conceptual, la evaluación es un término susceptible de diferentes definiciones y a menudo intercambiable por otros en el ámbito educativo: medición, notas, pruebas, juicio de valor, rendición de cuentas, entre otras. La mayoría de los profesores y también los estudiantes asocian la evaluación con la valoración cuantitativa del rendimiento académico. No son pocos los que la siguen utilizando, sólo para categorizar a cada sujeto o programa en función de los resultados propios o en comparación a otros.

Tyler define la evaluación como el proceso surgido para determinar en qué medida han sido alcanzados los objetivos previamente establecidos. Años más tarde, el mismo autor empezó a prestar interés por la eficacia de los programas y el valor intrínseco de la evaluación para la mejora de la educación. Aparece un desencanto con la escuela pública y crece la presión por la rendición de cuentas (Whitford y Jones, 1998).

De esta forma, el concepto de evaluación amplía sus criterios valorativos. La evaluación educativa no sólo se relaciona con los resultados y mejora educativa, sino que se constituye en un proceso integrado de las acciones de enseñanza-aprendizaje, que debe satisfacer las necesidades y demandas de cada usuario.

Desde la reforma se define evaluación como un proceso que emite un juicio respecto de uno o más atributos de algo o alguien, fundamentado en información obtenida, procesada y analizada correctamente que a su vez es contrastada con un referente claramente establecido, sustentado en un marco de referencia consistente con él, que está encaminado 
a mejorar los procesos educacionales y que produce efectos educativos en sus actores involucrados, para lo que se apoya en el diálogo y la comprensión (Himmel, 1999).

Asumido el concepto y analizado desde el aprendizaje profesional, la evaluación, más que un instrumento para controlar y reflexionar acerca de los procesos y resultados, debe legitimar frente a docentes y la sociedad en general que lo aprendido es capaz por sí solo de justificar políticas públicas en la materia y dejar atrás la forma tradicional de combatir con programas transitorios, un problema que se ha convertido en permanente, como es el aislamiento y la desprofesionalización de los docentes (Hargreaves, 1998).

Desde su irrupción en el mundo educativo y formativo, la evaluación ha generado importantes expectativas no sólo de carácter pedagógico, sino también de carácter social y económico, lo que unido al creciente interés por la calidad educativa en cualquiera de sus manifestaciones y ámbitos hace que se imponga la necesidad de desarrollar modelos de evaluación adecuados al objeto y a los distintos contextos en los que se produce.

La literatura internacional señala la existencia de tres enfoques de evaluación, orientados a la toma de decisiones respecto de los programas de formación permanente (Jiménez, 1999), a saber:

a) El enfoque para la mejora, que orienta sus investigaciones a evidenciar la adecuación de los planes de estudio, la atención al currículo, los métodos de enseñanza, la coordinación y los procedimientos evaluativos; para ello busca mecanismos que apoyen el control externo, los incentivos, el liderazgo y colegiabilidad del profesorado, además de normar los procesos de gestión y la coordinación de los implicados. Su objetivo es mejorar la gestión del servicio.

b) El enfoque para la autorregulación, que busca estudiar los planes y planificaciones, los sistemas de control interno y procedimientos externos y la participación de los docentes en la gestión de los programas con el propósito de decidir respecto de la satisfacción de los docentes, la evaluación externa y el seguimiento postintervención. $\mathrm{Su}$ objetivo es consolidar los procedimientos que posibilitan el aseguramiento de la calidad de los programas.

c) El enfoque de la eficacia, que se orienta a evaluar el impacto de los programas según el cumplimiento de sus objetivos, metas, rendimiento de los docentes, su reinserción profesional y la concreción de los aprendizajes de los docentes y estudiantes, a fin de establecer estándares de calidad.

Discutiendo el aporte de tales perspectivas, se puede apreciar que la evaluación -ya sea para mejorar, como autorregulación o de la eficacia- enfatiza el papel de la audiencia, de la evaluación y de la relación del evaluador con ella. Así, tal como lo sugieren los estudios previos de los postítulos (Cancino, et al., 2006; González y Trebilcock, 2008), la audiencia son los actores involucrados, los docentes y las acciones. Además, la evaluación no puede realizarse sin un análisis de las variables de procedencia (género, experiencia, entre otros) y las características estructurales (duración, participación colectiva) que condicionan el impacto de tales instancias (Ingvarson, 2002). Finalmente, los principales enfoques de evaluación de la formación permanente olvidan referirse a aspectos importantes. Aunque cada perspectiva evalúa una dimensión importante de la formación, existe poco diálogo entre ellos. Y aunque se esfuerzan en operacionalizarlas, sólo la perspectiva de la eficacia permite abordar mejor el problema planteado. Al ser 
un enfoque explicativo, capaz de proporcionar las respuestas necesarias para comprender al docente, no como sujeto pasivo de la evaluación, sino como un informante clave para evaluar el impacto de los programas en su saber pedagógico. Como enfoque, proporciona un marco para estudiar el modo en que las características estructurales y de proceso inciden en la eficacia de la formación permanente.

\section{I.2. EVALUACION “LA EFICACIA DE LA FORMACION PERMANENTE: PERSPECTIVA DEL ESTUDIO}

La aproximación evaluativa del proyecto está basada en una revisión de los estudios recientes sobre las características de los programas de formación permanente eficaces (Kennedy, 1998; Loucks-Horsley et al., 1998; Wilson y Valli, 1999; Garet et al., 2001; Supovitz, 2001; Guskey y Sparks, 2002; Miranda, 2005; Ingvarson, 2005). Esta investigación ha llegado a ser cada vez más sofisticada durante estos años, y otorga bases firmes para desarrollar un modelo con el cual contar para la mayoría de las diferencias en la efectividad de programas de formación permanente. Este modelo avanza en la perspectiva de Stake, quien propone un método de evaluación centrado en la satisfacción del usuario del programa o "respondente" (Stake, 1975 y 1975a), asumiendo que los objetivos de los programas deben responder a los problemas y cuestiones reales que plantean los implicados. Según Stufflebeam y Shinkfield (1987), este modelo hace de Stake un referente de una nueva escuela de evaluación, que exige un método orientado al servicio y la medición de la eficacia. El autor distingue tres aspectos a evaluar en los programas: i) antecedentes, es decir, cualquier condición existente antes de la actividad formativa que pueda relacionarse con los resultados. Como por ejemplo, las variables de procedencia (ej. edad, años de experiencia, sector de aprendizaje y nivel escolar); ii) transacciones, como las características estructurales e interacciones que se producen entre las actividades y las oportunidades para aprender de los docentes, y iii) impactos inmediatos (ej. cambio en los conocimientos, eficacia y práctica mejorada) y a largo plazo (ej. aprendizaje estudiantil).

Como se observa, este modelo está en la base de las investigaciones recientes sobre las características de programas de formación permanente eficaces (Garet et al., 2001; Ingvarson, 2005), al centrar su mirada en las interacciones altamente asociadas a la eficacia de los programas como son las características (a) estructurales y (b) de proceso.

a) Estructurales: se pueden caracterizar según: Duración. Consistentemente la literatura reciente sobre el aprender del profesor y la formación permanente señala que para que el desarrollo profesional se logre, se debe dar en un cierto plazo. Se espera que la duración de las actividades de formación sea importante de dos maneras. Primero, se asume que las actividades más duraderas proporcionan más oportunidades para la discusión profundizada del contenido, las creencias y teorías implícitas de los docentes y las estrategias pedagógicas. En segundo lugar, las actividades que se extienden en un cierto plazo, probablemente, permitan que los profesores prueben nuevas prácticas en la sala de clases y que obtengan la regeneración en su enseñanza; Participación colectiva. Hay un interés cada vez mayor en el diseño de estrategias de formación para grupos de profesores pertenecientes a una misma escuela, nivel o disciplina. El diseño de programas para grupos de profesores pares de una misma unidad tiene un número de ventajas potenciales. Primero, los profesores que trabajan juntos, o 
son de una misma especialidad, tienen más oportunidad de discutir conocimientos, habilidades y problemas que se presentan durante sus experiencias de formación permanente. En segundo lugar, los profesores que son de la misma escuela, comuna o grado probablemente compartan los mismos objetivos y contenidos educacionales, las características socioculturales de sus estudiantes y los requisitos comunes de desempeño profesional; integrando con ello lo que aprenden con otros aspectos de su contexto educacional. Tercero, los profesores que laboran con los mismos estudiantes pueden discutir las necesidades de éstos a través de clases y reflexiones colectivas. Para Ball (1995), la participación colectiva en la misma actividad puede proporcionar un foro para la discusión y el entendimiento, aumentando la capacidad de los profesores de crecer. Existe poca información acerca de la incidencia de acercamientos colectivos para el desarrollo profesional, pero hay una cierta evidencia de que este trabajo entre pares puede ser eficaz en el cambio de la práctica. Newmann (1996), en un estudio de 24 escuelas, observa que la formación permanente centrada en grupos de los profesores de matemáticas de la misma escuela o comunidad mejoraban las habilidades disciplinares de éstos al compartir prácticas eficaces.

b) De Proceso: Se puede estructurar según si están: Focalizadas en el Contenido. Aunque hay abundante literatura sobre la formación permanente, es asombrosa la poca atención que se ha dado a lo que aprenden los profesores realmente en las actividades de desarrollo profesionales, es decir, su contenido. En particular, poca investigación se ha conducido sobre la eficacia relativa de las actividades de formación permanente que se centren en diversos tipos de creencias, conocimiento y de prácticas de enseñanza (Kennedy, 1998). La investigación descriptiva disponible sugiere que el contenido tratado durante actividades de formación permanente varía en cuatro dimensiones: i) el énfasis que dan al tema, que se espera los profesores apropien y enseñen en la práctica; ii) las actividades que se planifican para mejorar el conocimiento del contenido de los profesores del tema o disciplina; iii) el diseño didáctico para mejorar la pedagogía general o la práctica de enseñanza, tales como liderazgo en la sala de clase, y iv) mejorar lo que Shulman (1987) ha llamado el conocimiento didáctico del contenido pedagógico en contextos específicos. Puesta en práctica. Aunque hay poca evidencia sobre la eficacia de las actividades de formación permanente en conocimiento, práctica y competencias de enseñanza, ha emergido un grupo de estudiosos que ha centrado su investigación en las actividades. En tales estudios se sugiere que aquel programa de formación permanente que se centre en actividades prácticas sobre el tema-materia y cómo los niños aprenden, puede ser un elemento especialmente importante en la práctica de enseñanza que cambia (Corcoran, 1995). Esta discusión es relevante en el caso de los hallazgos sobre los profesores de básica en Chile que carecen de seguridad y habilidades en la enseñanza de contenidos específicos en Matemáticas y Ciencias, tal como lo refleja el TIMMS de 1999 (MINEDUC, 2005) y que pretenden ser intervenidos mediante programas como el PPM, PPC. En la misma dirección Reynolds (1995: 214), en una revisión del conocimiento disciplinar (ciencias) de profesores de primaria en EE.UU. concluía que los "profesores que comienzan tienen asombrosamente poco entendimiento pedagógico del contenido específico". A su vez Rin (1998:27), en una discusión del papel de la investigación en la enseñanza, precisa que "los profesores 
tienen necesidad de formación permanente que proporcione maneras más prácticas de tratar el contenido". Oportunidades para el aprendizaje activo. Se refiere a las oportunidades proporcionadas por la actividad para que los profesores se integren activamente a la discusión significativa, el planeamiento y la práctica (Liberman, 1996). Las oportunidades para aprender activamente pueden tomar distintas formas, incluyendo la oportunidad de observar a profesores expertos; para planear cómo los nuevos materiales del plan de estudios y métodos de enseñanza serán utilizados en aula; para repasar el trabajo del estudiante en los contenidos que son cubiertos, y para conducir discusiones de trabajos escritos (Darling-Hammond, 2004).

A partir de todo lo anterior, la aproximación evaluativa del estudio en los dos programas de formación permanente (PPMAT y PPECN) estará basada en un modelo causal formal de las principales características que podrían explicar las variaciones de los impactos que éstos generan. El marco está basado en una revisión de recientes investigaciones centradas en las características de programas de desarrollo profesional efectivo, principalmente en el enfoque evaluativo de Stake (1975), los hallazgos de Garet et al. (2001) en el contexto del Programa Eisenhower, Ingvarson (2002 y 2005) y sus estudios sobre el Programa de Calidad del Profesor del Gobierno Australiano y Miranda (2004, 2005 y 2006) en sus estudios sobre el impacto de la formación permanente en Chile.

La Figura 1 distingue los cuatro tipos de impacto que contempla el Modelo hipotético y que serían el resultado de los programas de formación permanente, considerados en el estudio. Estos incluyen el impacto en el conocimiento, la eficacia, la práctica y el aprendizaje estudiantil. El modelo también incorpora variables de procedencia, características estructurales, oportunidad para aprender y, como actor mediador, la comunidad profesional.

\section{Figura 1}

Modelo Hipotético del estudio

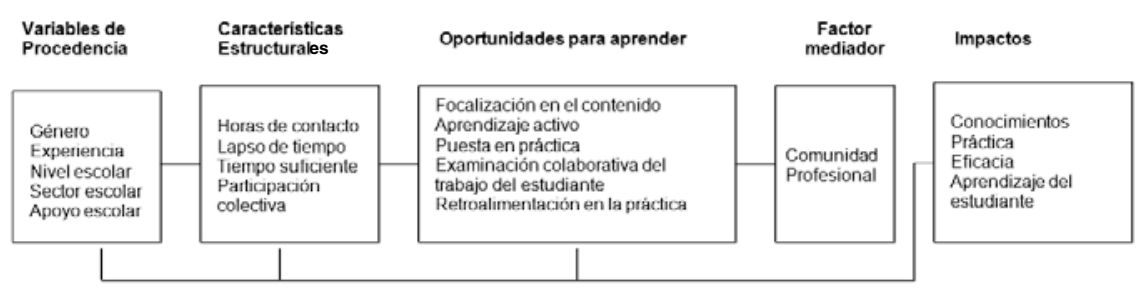

(Miranda, 2006).

En resumen, se trata de una nueva búsqueda de sentido, a la vez de continuidad y ruptura con las prácticas investigativas referidas a la eficacia de la formación permanente, con el fin de contribuir al debate sobre el lugar que ocupa el profesor en la sociedad, pero también con el propósito de dar una respuesta al problema de evaluar qué aprende y qué hace al desarrollo profesional eficaz, para determinar si su impacto amerita el costo respecto a otras prioridades para la política del fortalecimiento de la profesión docente en el actual contexto histórico y educativo. De lo anterior se desprende que todo estudio 
referido al examen de la eficacia de la formación permanente, en términos de impacto pedagógico (conocimiento, eficacia y práctica) y aprendizaje estudiantil, es necesario para comprender cómo cambian y se relacionan estos constructos según variables de procedencia, características de proceso, factores mediadores y oportunidades para aprender. Esta es la perspectiva y propósito de la presente investigación.

\section{METODOLOGIA}

La contrastación del Modelo Conceptual es operacionalizado en la escala ICAP, adaptada y validada en la primera etapa de la investigación, en 25 estudios de evaluación (Miranda, 2007). Estos estudios corresponden a los PPMAT (9), PPECN (9) y Grupo Control (7), siendo estos repeticiones del mismo modelo de formación. En enero de 2008 se cursó una invitación a la totalidad de universidades tradicionales que se adjudicaron propuestas en ambos PP en su versión 2007 de todo el país, para la jornada de trabajo en que el investigador y su equipo dieron a conocer los objetivos del proyecto y aplicarían los instrumentos según consentimiento informado y señalando la eventual posibilidad de asistir a sus unidades educativas de origen en la tercera etapa del estudio. Al finalizar la jornada se invitó a los coordinadores de las universidades a generar espacios de socialización de los resultados en caso de ser requeridos. Del total de universidades (12), todas respondieron, eliminándose aquellas que no ejecutaron propuestas por razones de acreditación de sus pedagogías o baja cobertura de matrícula (U. de Tarapacá, U. de Magallanes y U. de Atacama) $)^{2}$. La tabla 1 contiene los datos referentes a la muestra del estudio, una vez depurados según criterios de representatividad estadística (fracción de muestreo) y teóricas (género, heterogeneidad geográfica, entre otras).

Tabla 1

Distribución de la Muestra Control, PPMAT y PPECN finales por Género

\begin{tabular}{|l|c|c|c|c|c|c|}
\cline { 2 - 7 } \multicolumn{1}{c|}{} & \multicolumn{2}{c|}{ Grupo PPMAT } & \multicolumn{2}{c|}{ Grupo PPECN } & \multicolumn{2}{c|}{ Total } \\
\cline { 2 - 7 } \multicolumn{1}{c|}{} & $\mathrm{n}$ & $\%$ & $\mathrm{n}$ & $\%$ & $\mathrm{n}$ & $\%$ \\
\hline Hombres & 69 & 32,9 & 26 & 16,5 & 129 & 22,7 \\
\hline Mujeres & 141 & 67,1 & 132 & 83,5 & 439 & 77,3 \\
\hline Total & 210 & 100,0 & 158 & 100,0 & 568 & 100,0 \\
\hline
\end{tabular}

Los sujetos de la muestra son docentes en servicio, que laboran en escuelas municipales y particulares subvencionadas provenientes de todo el país, en su mayoría de sexo femenino y que aceptaron voluntariamente formar parte de esta investigación. Para la equivalencia inicial se contrastaron los datos con la población de docentes graduados en

2 Zona Norte: U. de Antofagasta y U. Católica de Valparaíso; Zona Centro: U. de Chile, UMCE, U. Católica del Maule; Zona Sur: U. de Concepción, U. Bío-Bío, UFRO y U. Católica de Temuco. 
la cohorte $2007(\mathrm{~N}=638)$, estudios nacionales (Miranda, 2005) e internacionales (Garet et al., 2001; Ingvarson, 2002 y 2005; Day, 2007), con lo cual la garantía de confianza de los resultados es mayor. Estos procedimientos permiten aseverar la representatividad de la muestra $(57,7 \%)$ y un testeo más riguroso del Modelo Conceptual cuyos resultados más importantes se presentan a continuación.

\section{RESULTADOS}

En este apartado se sintetizan los principales resultados derivados del estudio empírico de los datos. En éste se muestran desde los análisis exploratorios hasta los descriptivos por variable, pasando por el comparativo relacionado con los casos o grupos.

Tabla 2

Relación entre factores estructurales de los PP y el impacto medido en los Programas

\begin{tabular}{|c|c|c|c|c|c|c|c|c|c|c|}
\hline \multirow[t]{2}{*}{ PPMAT } & \multicolumn{2}{|c|}{ Comunidad } & \multicolumn{2}{|c|}{ Conocimiento } & \multicolumn{2}{|c|}{ Práctica } & \multicolumn{2}{|c|}{ Aprendizaje } & \multicolumn{2}{|c|}{ Eficacia } \\
\hline & Beta & Sig. & Beta & Sig. & Beta & Sig. & Beta & Sig. & Beta & Sig. \\
\hline Lapso de Tiempo & $-0,15$ & 0,18 & $-0,09$ & 0,42 & $-0,06$ & 0,96 & $-0,13$ & 0,28 & 0,01 & 0,86 \\
\hline Tiempo Suficiente & $-0,35$ & 0,02 & 0,26 & 0,31 & $-0,13$ & 0,22 & $-0,58$ & 0,58 & $-0,11$ & 0,33 \\
\hline Foco Contenido & 0,27 & 0,00 & 0,86 & 0,00 & 0,71 & 0,00 & 0,43 & 0,00 & 0,52 & 0,00 \\
\hline Aprend. Activo & 0,48 & 0,00 & 0,34 & 0,00 & 0,51 & 0,00 & 0,62 & 0,00 & 0,42 & 0,00 \\
\hline Ex. Colaborativo & 0,43 & 0,00 & 0,22 & 0,01 & 0,34 & 0,00 & 0,53 & 0,00 & 0,31 & 0,00 \\
\hline Retroalimenta & 0,47 & 0,00 & 0,22 & 0,02 & 0,37 & 0,00 & 0,47 & 0,00 & 0,35 & 0,00 \\
\hline Seguimiento & 0,18 & 0,01 & 0,08 & 0,36 & 0,80 & 0,35 & 0,16 & 0,08 & 0,06 & 0,50 \\
\hline Comunidad & & & $-0,76$ & 0,45 & 0,43 & 0,00 & 0,50 & $0,00^{*}$ & 0,37 & 0,00 \\
\hline $\mathrm{R}$ (ajustado) & \multicolumn{2}{|c|}{0,64} & \multicolumn{2}{|c|}{0,60} & \multicolumn{2}{|c|}{0,47} & \multicolumn{2}{|c|}{0,38} & \multicolumn{2}{|c|}{0,37} \\
\hline \multirow[t]{2}{*}{ PPECN } & \multicolumn{2}{|c|}{ Comunidad } & \multicolumn{2}{|c|}{ Conocimiento } & \multicolumn{2}{|c|}{ Práctica } & \multicolumn{2}{|c|}{ Aprendizaje } & \multicolumn{2}{|c|}{ Eficacia } \\
\hline & Beta & Sig. & Beta & Sig. & Beta & Sig. & Beta & Sig. & Beta & Sig. \\
\hline Lapso de Tiempo & 0,58 & 0,71 & $-0,06$ & 0,88 & $-0,42$ & 0,05 & $-0,21$ & 0,18 & 0,13 & 0,62 \\
\hline Tiempo Suficiente & $-0,30$ & 0,02 & $-0,08$ & 0,87 & 0,43 & 0,64 & 0,07 & 0,85 & $-0,23$ & 0,12 \\
\hline Foco Contenido & 0,46 & 0,00 & 0,66 & 0,00 & 0,85 & 0,00 & 0,72 & 0,00 & 0,73 & 0,00 \\
\hline Aprend. Activo & 0,73 & 0,00 & $-0,37$ & 0,05 & 0,37 & 0,00 & 0,48 & 0,00 & 0,37 & 0,00 \\
\hline Ex. Colaborativo & 0,81 & 0,00 & 0,53 & 0,06 & 0,36 & 0,00 & 0,48 & 0,00 & 0,40 & 0,00 \\
\hline Retroalimentación & 0,87 & 0,00 & $-0,01$ & 0,96 & 0,33 & 0,00 & 0,44 & 0,00 & 0,36 & 0,00 \\
\hline Seguimiento & $-0,10$ & 0,35 & 0,17 & 0,27 & 0,18 & 0,08 & $-0,22$ & 0,84 & 0,11 & 0,30 \\
\hline Comunidad & & & 0,22 & 0,02 & 0,43 & 0,00 & 0,46 & 0,00 & 0,43 & 0,00 \\
\hline R (ajustado) & \multicolumn{2}{|c|}{0,67} & \multicolumn{2}{|c|}{0,65} & \multicolumn{2}{|c|}{0,54} & \multicolumn{2}{|c|}{0,47} & \multicolumn{2}{|c|}{0,44} \\
\hline
\end{tabular}


Los análisis separados por programas (según área disciplinar) se constituyen en dos estudios independientes (cada uno fue desarrollado bajo procedimientos independientes) en su construcción pero complementarios a la línea de investigación del equipo, los que fueron agrupados siguiendo los criterios del Análisis de Regresión (Modelo Lineal General) conducido por SPSS Versión 12.0. Se basa en el cálculo de algoritmos cuadráticos orientados a estimar la fuerza de la relación lineal entre Variables Dependientes y un grupo de Variables Independientes, cuyo orden de ingreso fue según variables exógenas (Impactos) y endógenas (Procedencia, Características Estructurales, Oportunidades para aprender y Comunidad Profesional), siguiendo el Modelo Hipotético del estudio.

De la Tabla 2 se pueden observar los coeficientes de regresión y niveles de significatividad estandarizados. De ellos se extrae la comparación entre la fuerza de la relación en cada modelo. Ejemplo Beta de Aprendizaje Activo es 0,48 es casi tres veces más fuerte que 0,18 de Seguimiento en la variable Conocimiento en los PPMAT.

El modelo permite examinar los impactos desde una red de efectos, lo cual constituye una contribución relevante al responder, mediante la regresión, cómo cada variable contribuye de manera individual a la variable dependiente considerada. Esto último, en una perspectiva global, permite una amalgama de resultados a través de los dos estudios de evaluación, constitutivos del proyecto común. Es importante señalar que los dos modelos responden a un mismo "experimento", esto es, el Programa de Postítulo de Mención. En tal sentido son réplicas que comparten los mismos objetivos políticos y supuestos respecto a su necesidad. Esto permite aseverar que, al ser el análisis una amalgama de resultados basados en un mismo instrumento y cuyos sujetos de análisis responden a un mismo tipo de programa, tales resultados pueden ser considerados de gran confiabilidad estadística.

Se ha omitido en este primer análisis al Grupo Control, por estar sus datos en fase de depuración.

Sumado lo anterior, a los indicadores de fiabilidad reportados sobre el instrumento (Ingvarson, 2005; Miranda, 2007) se puede argumentar en torno al cumplimiento de las tareas propuestas referidas a la contrastación del Modelo Conceptual sobre los factores que afectan el impacto de los Programas de Postítulo sobre el desarrollo profesional docente, situación que posiciona al estudio en una primera línea en el ámbito de la investigación sobre el desarrollo profesional eficaz.

De este modo, la relación entre las variables estructurales de procedencia, características del proceso, las propuestas de desarrollo profesional, oportunidades de aprendizaje activo, seguimiento, medidas de logro, retroalimentación, eficacia y mejoramiento en la práctica y el conocimiento constituyen elementos centrales de una propuesta de indicadores para la construcción de estándares de calidad de la formación permanente de profesores.

A partir de lo anterior, el análisis se focaliza en las variables de impacto, a saber: a) conocimiento, b) práctica, c) aprendizaje estudiantil y d) eficacia.

a) Conocimiento. El foco del contenido en ambos programas aparece como una variable fuertemente relacionada a esta medida de impacto. Así, en el caso del Programa PPMAT 0,60 y en el PPECN 0,65, ambos con un nivel de significatividad alto $(\mathrm{p}=0,01)$. Además, la relación entre la variable y la Comunidad Profesional es inversa $-0,76$ y poco significativa a nivel $(p=0,45)$. Un dato duro relevante es la presencia 
de diferencias importantes entre los PP en la variable predictora Aprendizaje Activo: mientras el PPMAT es alta y positiva 0,34 y significativa $(\mathrm{p}=0,01)$ en el PPECN es baja y negativa $-0,37$ y significativa $(p=0,05)$. Finalmente, el modelo estaría evidenciando el bajo valor de las variables asociadas al tiempo de aprendizaje profesional como factores predictores del impacto de los PP en la variable de estudio.

b) Práctica. En ésta el impacto de los PP es mediado por las variables asociadas al bloque Oportunidades para Aprender y Comunidad Profesional. En ambos programas las variables asociadas al primer bloque se presentan como predictores con una relación positiva y significativa y, en el segundo, de igual modo, siendo la dimensión Foco del Conocimiento la más alta en ambos casos: PPMAT 0,71 y PPECN 0,85. Además, el bloque de Características Estructurales aparece con valores diferentes, siendo bajos y negativos en ambos casos la dimensión Lapso de Tiempo y distintos en Tiempo Suficiente: mientras en el PPECN es positivo y significativo $(\mathrm{p}=0,05)$ en el PPMAT es bajo y negativo y poco significativo. Finalmente, el modelo es constante en su predicción para ambos programas, en PPMAT 0,47 y en PPECN 0,54.

Al relacionar las medidas de impacto anteriores con la variable mediadora Comunidad Profesional ésta aparentemente está actuando como articuladora en ambos programas, reafirmando su relevancia en el Modelo Conceptual propuesto. Un ejemplo de ello es cuando los docentes de la muestra responden masiva y positivamente a la pregunta sobre si el Programa acrecienta su apoyo y refuerza o mejora los niveles de profesionalismo en la Comunidad Escolar. Al parecer, el focalizar su mirada en sus prácticas de enseñanza y las oportunidades de aprendizaje de los estudiantes, compartir ideas y apoyarse entre pares es una forma de implementar y transferir al aula "nuevas ideas", generadas en los Programas. De este modo, el alcance de los Postítulos estaría influenciando el nivel de mediación en la Comunidad Profesional y mejorando con esto el diseño de procesos de construcción de conocimientos, seguimiento y oportunidades de examinación colaborativa del trabajo de los estudiantes.

c) Eficacia. La tabla 1 presenta la proporción de la varianza explicada por el Modelo $\left(\mathrm{R}^{2}\right)$ en ambos programas estudiados según esta medida de impacto. Aunque hay variabilidad en la varianza encontrada se puede afirmar su consistencia en cada uno de ellos. De este modo el Modelo explica en los Programas: PPMAT (37\%) y en el PPECN (44\%), C (45\%). Esto significaría que muchas características del Modelo propuesto son razonablemente buenos predictores. Esto se explicaría por el hecho que los profesores ven en la formación permanente un factor clave de su desarrollo profesional efectivo. Además, nuevamente el bloque Características Estructurales tiene una baja ponderación como predictor del impacto en ambos programas, donde la variable Tiempo Suficiente se mantiene como una dimensión negativa y poco significativa. Por otro lado, las variables Foco del Contenido y Aprendizaje Activo son elementos centrales del peso del bloque Oportunidades para Aprender en ambas propuestas. No obstante, la dimensión Seguimiento en el PPMAT $(0,06)$ y PPECN $(0,11)$ es la más baja, lo cual es una constante en la mayoría de las variables de impacto, lo cual se puede deber a su ausencia permanente en los diseños lógicos y de acción documentados en la primera etapa del estudio. Esto último se constituye 
en un nudo crítico de los PP en particular. Finalmente, la Comunidad Profesional es un predictor estable y constante en el PPMAT y PPECN, situación documentada en la literatura internacional (Ingvarson, 2005) como un elemento centrado en la posibilidad y habilidad que tenga el docente beneficiario para replicar y transferir sus nuevos saberes a nivel de colegio y aula.

d) Aprendizaje estudiantil. Al contemplar esta medida de impacto con el las variables del bloque Oportunidades para Aprender, el Foco del Contenido y el Aprendizaje Activo nuevamente se observa una fuerte relación en ambos programas. Además, el Seguimiento nuevamente está poco relacionado con el aprendizaje estudiantil según la percepción de los profesores encuestados. Asimismo, el Examen Colaborativo actúa de manera significativa sobre la variable. Por otro lado, el Bloque Características Estructurales se relaciona de manera negativa y baja con el aprendizaje estudiantil, siendo una excepción a esta regla el Tiempo Suficiente en el PPECN que lo hace de manera positiva pero leve $(0,07)$. Finalmente, la Comunidad Profesional actúa de manera importante y positiva sobre esta variable. Sin duda, este resultado refuerza el rol de los líderes escolares y profesores pares que no participan en la actividad de desarrollo profesional como actores clave en la seguridad y capacidad que el docente beneficiado pueda tener para aplicar lo aprendido en su escuela.

De lo anterior se puede aseverar que las Actividades de Aprendizaje Activo aparecen como una variable particularmente relevante. Al constituirse en una acción que está a la base de una red de influencias. Los resultados de este estudio y los de Ingvarson (2005) son consistentes al respecto, sugiriendo con ello que esta característica de proceso tiene efectos penetrantes y genera influencia en los factores que incrementan la confianza de los profesores y su habilidad para responder a las necesidades de aprendizaje de los estudiantes, más que provocar cambios específicos en sus prácticas pedagógicas. Esta variable en el Modelo Conceptual propuesto tiene efectos directos en el Conocimiento del profesor. Así como el Conocimiento del profesor fue hipotetizado, los resultados serán contrastados mediante un análisis que permita relacionarlos con las otras variables de impacto, a saber: Práctica, el Aprendizaje de los Estudiantes y la Eficacia percibida.

Uno de los mayores descubrimientos de este estudio es que sobre todas las variables del modelo el Bloque de Oportunidades de Aprendizaje tiene un efecto notable en los logros de los Programas de manera individual. Si se observa el impacto de estas variables hay patrones emergentes de razonable consistencia, tal como se describiera para el caso del Conocimiento y el resto de las variables de impacto.

Se constata baja variabilidad y alta consistencia en la incidencia de las características estructurales y de proceso en el impacto del PPMAT y PPECN en las variables de resultado. El modelo completo dio cuenta de casi el $38 \%$ de la discordancia en la variable eficacia del docente en el PPMAT y $46 \%$ en el PPECN, lo cual significa que un conjunto importante de factores del Modelo Conceptual son predictores fiables sobre la eficacia de los programas, según la percepción de los docentes beneficiarios. Los factores asociados al bloque Oportunidades para Aprender tuvieron el mayor efecto en los resultados individuales según programa por universidad. El factor que más influye en el 
impacto de los PPMAT y PPECN en el Conocimiento es el Foco del Contenido, con un Beta de 0,86 y 0,66, respectivamente. En la Práctica los factores que más afectan son el Foco del Contenido, Aprendizaje Activo y Examen Colaborativo. En el primer caso, en el PPMAT es $(0,71)$ y PPECN $(0,85)$, en el segundo $(0,51)$ y $(0,37)$ y en el último $(0,34)$ y $(0,36)$, respectivamente. En la Eficacia observada en el PPMAT y PPECN inciden el Foco del Contenido $(0,52)$ Aprendizaje Activo $(0,42)$ y retroalimentación $(0,35)$ en el primero y de $(0,73),(0,37)$ y $(0,40)$ en el segundo. En el Aprendizaje Estudiantil inciden de manera decreciente: Aprendizaje Activo $(0,62)$ y Foco del Contenido $(0,43)$ en el PPMAT, situación que se itera en orden inverso en el PPECN, siendo el primero $(0,48)$ y $(0,72)$ el segundo.

Al observar el impacto de los programas en las variables de resultado juntas, la importancia de la Comunidad Profesional como variable mediadora se hace evidente. En efecto, los resultados indican que las relaciones entre la Variable Mediadora y los niveles informados del impacto en Conocimiento, Práctica, Aprendizaje Estudiantil y Eficacia son significativas en ambas propuestas. La magnitud con que la variable incide en la Práctica (0,43), Aprendizaje Estudiantil $(0,50)$ y Eficacia $(0,37)$ en el PPMAT y $(0,43),(0,46)$ y $(0,43)$ en el PPECN, respectivamente, de acuerdo a la percepción de los docentes encuestados, realza hasta qué punto las propuestas refuerzan la influencia de la Comunidad Profesional (Directivos, pares) en la eficacia de la formación permanente en la escuela; esto es, el grado con el cual aumentan las posibilidades para los profesores para compartir ideas, colaborar en la evaluación de los trabajos de sus estudiantes y recibir retroalimentación por parte de su pares que no participaron en los Programas de Postítulo sobre la Práctica de sus aprendizajes a nivel de saber pedagógico. La fuerza con que la Comunidad Profesional R $(0,36)$ media en el impacto de los Programas debería ser considerada al momento de la mejora de las propuestas, en especial en lo referido a las variables de resultado Práctica $(0,30)$, Aprendizaje Estudiantil $(0,38)$ y Eficacia $(0,31)$.

Hubo diferencias en el grado en el cual los PPMAT y PPECN incorporan las variables de Oportunidades para Aprender en la puesta en práctica de sus propuestas. En una escala de 1 a 4, la Media Aritmética fue cercana a 4 para la Focalización del Contenido; 3,5 para el Aprendizaje Activo; 3,3 para la Examinación Colaborativa, 3,0 en Retroalimentación y menor a 2 en el caso del Seguimiento. Pese a esta diferencia de énfasis, en la literatura internacional se reporta una fuerte relación entre ellas (Garet, et al., 2001; Ingvarson et al., 2005). Por otro lado, los resultados sugieren que en general, los PPMAT tienen mayor énfasis que los PPECN no sólo en el tema que se está enseñando, sino también en cómo se está aprendiendo y cómo es enseñado, tendiendo a facilitar de una manera más activa los procesos de formación permanente efectivos. Al contrastar la evidencia cualitativa sobre el enfoque teórico basal de las propuestas y su estrecha relación con la variable Universidad, este estudio avanza en la interpretación referida a que los PP que conceptualizan desde el Modelo de Desarrollo Profesional y enfoque de Desarrollo y Mejora construyen oportunidades para el apoyo, no sustitución, de la práctica pedagógica, donde la valoración de la experiencia previa y retroalimentación de los formadores de profesores y pares con más experiencia constituyen elementos centrales en el impacto de las propuestas en las variables de impacto consideradas según la percepción de los docentes de la muestra. 
Tabla 3

Medias Aritméticas de PP en variable Aprendizaje Estudiantil según zona geográfica

\begin{tabular}{|l|c|c|c|c|c|c|c|c|}
\hline & \multicolumn{2}{|c|}{ Norte } & \multicolumn{2}{c|}{ Centro } & \multicolumn{2}{c|}{ Sur } & \multicolumn{2}{c|}{ Total } \\
\hline Puntajes & PPMAT & PPECN & PPMAT & PPECN & PPMAT & PPECN & media & $\mathrm{n}$ \\
\hline Alto & 3,7 & 3,7 & 3,5 & 3,7 & 3,7 & 3,7 & 3,6 & 6 \\
\hline Bajo & 2,8 & 2,8 & 2,0 & 2,8 & 1,7 & 1,2 & 2,2 & 6 \\
\hline Diferencia & 0,9 & 0,9 & 1,5 & 0,9 & 2,0 & 2,5 & 1,4 & 12 \\
\hline
\end{tabular}

La tabla 3 presenta a docentes de altos y bajos puntajes promedio en la variable Aprendizaje Estudiantil asociados al PPMAT y PPECN, según Zona Geográfica. De los datos se puede decir que los profesores que tienen un puntaje cercano al 4 perciben estar "Completamente de acuerdo" en que el PP enfatiza y tiene impacto en esta variable de resultado. Caso contrario para el puntaje promedio cercano al 2 los docentes manifiestan estar "En desacuerdo" sobre la misma situación. Como se observa según grupo por zona, hay una estabilidad relativa entre los puntajes extremos, siendo más alta la diferencia en la Zona Sur y menores en Norte y Centro. Además, se pueden constatar leves diferencias promedio según programa, siendo relevante este dato para la equivalencia inicial del estrato, tal como lo exige el diseño del estudio original (Miranda, 2006).

\section{A MODO DE CONCLUSION}

Este artículo ha cumplido los objetivos propuestos. El mensaje principal es que entre todas las variables en el Modelo Conceptual el bloque de Oportunidades de Aprendizaje tienen un efecto relevante en cada una de las propuestas, situación que se cruza teóricamente con los modelos basales de los PP, donde la variable Foco del Contenido y Aprendizaje Activo actúan como catalizadores de propuestas orientadas a la Capacitación o al Desarrollo Profesional. Finalmente, el hecho que la Comunidad Profesional influencie la práctica del profesor, su eficacia y en especial el Aprendizaje Estudiantil, es particularmente significativo. Esto estaría sugiriendo que, por un lado, los PP cumplen su objetivo central relativo a optimizar el Conocimiento del contenido y, por otro, que profesores pares y líderes de los colegios de procedencia tendrían una influencia penetrante y decisiva en los factores que inciden en la confianza, puesta en práctica y posible transferencia que los docentes beneficiarios tienen para satisfacer las necesidades de aprendizaje de sus estudiantes y, por ende, en el mejoramiento de la calidad educativa.

\section{BIBLIOGRAFIA}

Adelman, N. (1998). Teachers, time and school reform. In A. Hargreaves (Ed.), ASCD Yearbook, Rethinking educational change with heart and mind (pp. 92-100). Virginia: Asssociation for Supervisión and Curriculum Development. 
Aguerrondo, I. (2004). Mecanismos de control de calidad de la formación docente. Montevideo: ICET.

Alvarado, L. (Compilador). (2003). Formación de profesores en América Latina: Diversos contextos sociopolíticos (pp. 1-17). Bogotá. Ediciones Antropos.

Angulo, F. (2004). La evaluación de la formación permanente del profesorado en la Comunidad Autónoma de Canarias. Documento de Trabajo. Sevilla: Universidad de Sevilla.

Avalos, B. (2002). Profesores para Chile, Historia de un proyecto. Santiago: MINEDUC.

Ball, D. (1995). Which and eye on the mathematical horizon: Dilemmas of teaching elementary school mathematic, Elementary School Journal, 93, 373-398.

Ball, D. (1996). Teacher learning and the mathematic reform. What we think when know and what we need to learn. Phi Delta Kappa 77 (7) 500-508.

Biddle, B., Good, T. \& Goodson, I. (1997). International Handbook of Teachers and Teaching (2 vols.). Dordrecht, Holanda: Kluwer Academic Publishers.

Bransford, J. (Ed.) (1999). How people learning: brain, mind, experience, and school. Washington, C.C.: Nacional Press.

Castillo, E. y Cerda, A. (2006). La formación continua de profesores con universidades chilenas. Una Alianza que dinamiza el sistema educativo. Santiago: CPEIP.

Cochran-Smith, M. \& Lytle, S. (1999). Relationships of Knowledge and Practice: Teacher Learning in Communities. Iran-Nejad y Pearson. Review of Research in Education (pp. 249-305). Washington, American Educational Research Association.

Corcoran, T. (1995). Transforming professional development for teachers: A guide for state policymakers. Washington: National Governors'Association.

Cox, C. (2003). El nuevo currículo del sistema escolar. Documento de Trabajo. Santiago: MINEDUC.

Christensen, D. (1996). The professional knowledge-research base for teacher education. Sikula, J. Buttery, T., E. Guyton (Eds.) Handbook of Research on Teacher Education (pp. 38-52). New York, Macmillan.

Darling-Hammond, L. \& Brandsford, J. (2005). Preparing Teachers for a Changing World: What Teachers Should Learn and Be Able to Do. Hardcover: The National Academic Education.

Darling-Hammond, L. (2004). El derecho de aprender. Crear buenas escuelas para todos. Barcelona: Ariel.

Del Valle, M. (2002). Técnicas cualitativas de investigación social. Madrid: Síntesis.

Domínguez, P. (1998). Perfeccionamiento docente y televisión educativa. Tesis Doctorado en Ciencias de la Educación. Santiago: PUC.

El Rhin, S. (1998). The role of research and teachers knowledge base in professional development. Educational Research, 27 (5), 27-31.

Fernández-Ballesteros, R. (1995). Evaluación de Programas. Una Guía Práctica en Ámbitos Sociales, Educativos y de Salud. Madrid: Editorial Síntesis.

Garet, M., Porter, A., Desimone, L., Birman, E. \& Joon, K. (2001). What makes professional development effective? Result From a National Sample of Teachers. American Educational Research Journal, 38 (3), 915-945.

Glasser, B. \& Strauss, A. (1967). The discovery of grounded theory. Chicago, Aldeline.

Gonzalez, M. (1999). Creencias, autotoeficiencia y atribuciones en profesores básicos municipalizados. Tesis Doctorado en Ciencias de la Educación. Santiago: PUC.

Guskey, T. \& Sparks, D. (2002). Linking professional development: a new consensus. In L. DarlingHammond y G. Sykes (Eds.). Teaching in the learning profession. Handbook on policy and practice (pp. 127-150). San Francisco: Jossey-Bass Publishers.

Hargreaves, A. (1998). International Handbook of Educational Change. Gran Bretaña: Kluwer.

Heller, J., Daehler, J. \& Shinihara, L. (2003). Conneeting all the pieces. Journal of Staff Development 24 (4), 36-41. 
Hernández, R., Fernández, C. y Baptista, P. (1991). Metodología de la Investigación. Madrid: McGraw-Hill.

Himmel, E. (1999). Hacia una evaluación Educativa. Santiago: MINEDUC.

Hurtado, E. (2001). Representaciones del profesor en la integración de la computación a las prácticas docentes. Tesis Grado de Doctor en Ciencias de la Educación. Santiago: PUC.

Imbernon, F. (1996). La formación y el desarrollo profesional del profesorado. Barcelona: Aula.

Infante, M. (1996). Evaluación de las pasantías de profesores en el extranjero. Santiago: MINEDUC.

Ingvarson, L. (1998). Teaching Standards: Foundations for Professional Development Reform. Hargreaves, A. International Handbook of Educational Change (pp. 1006-1031). Gran Bretaña: Kluwer.

Ingvarson, L. (2002). Building and learning professional. Commissioned Research Series (htpp:// hacer.edu.au/publications).

Ingvarson, L. (2005). Factors affecting the impact of professional development programs on teachers knowledge, practice, students outcomes y efficacy. Educational Policy Analysis Archives, Vol. 13 (10).

Jiménez, B. (1999). La evaluación de programas, centros y profesores. Madrid: Síntesis.

Kennedy, M. (1998). Form and substance in in-service teacher education research monograph (13). Arlington, VA: National Science F.

Killion, J. (2003). Solid Footwork makes evaluation of staff development a song. Journal of Staff Development 24 (4), 14-12.

Latorre, M. (2004). Estudio sobre las prácticas pedagógicas en docentes de Octavo Básico. Proyecto FONDECYT Posdoctorado. Santiago: Universidad Alberto Hurtado.

Lee, O. \& Yarger, S. (1996). Modes of Inquiry in Research on Teacher Education. J. Sikula, T. Buttery, E. Guyton (Eds.). Handbook of Research on Teacher Education (pp. 14-37). New York, Macmillan.

Liberman, A. (1996). Networks and Reform in American Education. Teacher College Record, Vol. $98, \mathrm{~N}^{\circ} 1$, pp. 7-45.

Loucks-Horsley, S., Hewson, P., Styles, K. (1998). Designing professional development for teachers of science and mathematics Thousand Oaks. C.A.: Corwin Press.

Malcolm, W. (2003). Making sense of social research. London: Sage.

Marcelo, C. (1999). Formación de profesores para el cambio educativo. Barcelona, EUB.

Marcelo, C. (2002). Aprender a Enseñar Para La Sociedad del Conocimiento. Educational Policy Analysis Archives, Vol. 10 (35).

Martínez, E. (2008). A este ritmo no llegamos. Revista Capital, 232 (28-30).

MINEDUC (2002). Seis años de una política de perfeccionamiento docente: evaluación programa de becas en el exterior para profesionales de la educación. Santiago: MINEDUC.

MINEDUC (2004). Estudio explicativo sobre resultados TIMMS-R. Santiago: MINEDUC.

MINEDUC (2005). Informe de la Comisión de Formación Inicial. Santiago: MINEDUC.

MINEDUC (2006). Docentes para el Nuevo Siglo. Santiago: MINEDUC.

Miranda, Ch. (2003). La formación docente en Chile: investigación, tendencias y práctica. En Alvarado, L. (Compilador). (2003). Formación de profesores en América Latina: Diversos contextos sociopolíticos. Cap. II (pp. 18-50). Bogotá. Ediciones Antropos.

Miranda, Ch. (2004). Impacto del Programa de Becas en Exterior sobre la autoestima profesional, el pensamiento crítico y la innovación en las prácticas pedagógicas de los docentes beneficiados. Tesis Doctorado en Ciencias de la Educación. Santiago: PUC.

Miranda, Ch. (2005). Formación permanente de profesores. Impacto en sus competencias profesionales. Santiago: Universidad Católica de Chile.

Miranda, Ch. (2006). Impacto del Programa de Becas en el Exterior sobre la innovación pedagógica y el aprendizaje estudiantil según los docentes beneficiados de la X Región. Informe de Avance. Valdivia: Universidad Austral de Chile. 
Miranda, Ch. et al. (2008). Concepciones sobre la formación permanente de profesores: una Mirada desde los programas de postítulo. Boletín de Investigación Educacional. Vol. 23. Santiago: PUC.

Newmann, F. (1996). Autentic achievement: restructuring eschool for intellectual quality. San Francisco: Jossey Bass.

Núñez, M. (2008). Metodología de enseñanza de las ciencias basada en modelamiento sociocognitivo y evaluación tridimensional de los Aprendizajes. Santiago: MINEDUC.

OCDE (2004). Informe del sistema educativo chileno. Santiago: MINEDUC.

OCDE (2009). Informe del Sistema educativo Superior de Chile. Santiago: MINEDUC.

Pérez-Jiménez, C. (2003). Formación de docentes para la construcción de saberes sociales. Revista Iberoamericana de Educación (33), pp. 37-55.

Pozo, I. (2006). Teorías cognitivas del aprendizaje. Novena Edición. Madrid: Morata.

Reynolds, A. (1995). The knowledge base for beginning teachers. Elementary School Journal, 95, 199-221.

Schulmann, L. (1987). Those Who Understand: Knowledge Growth in Teaching, Educational Researcher, Vol. 15, No 2, pp. (4-14).

Stake, R. (1975). La evaluación de programas, en especial la evaluación de réplica. En Dockrell, W. y Hamilton, D. Nuevas reflexiones sobre la investigación educativa. Madrid: Narcea.

Stake, R. (1975a). An approach to the evaluation of instructional programs. En Hamilton D. et al. (Ed.) Beyon the numbers game: A reader in educational Evaluation. London: McMillan.

Stufflembeam, D. y Shinkfield A. (1987). Evaluación sistemática. Guía teórica y práctica. Madrid: Paidós.

Supovitz, J. (2001). Traslating teaching practice into improved students achievement. F. Fuhrman (Ed.). From the capital to the classroom. Standards-bases reforms in the state. Part Two (8198). Chicago: University of Chicago.

Troncoso, A. (2003). Breve historia de la formación de profesores en Chile. En Alvarado. L. (Compilador). (2003). Formación de profesores en América Latina: Diversos contextos sociopolíticos. Cap. III (pp. 97-124) Bogotá. Ediciones Antropos.

Vaillant, D. (2004). Formación de docentes en América Latina. Reinventando el modelo tradicional. Barcelona: Octaedro.

Weiss, I., Montgomery, D., Ridgway, C. y Enlace, S. (1998). Local systemic change. NY: Research makes.

Wilson, S. \& Valli, J. (1999). Teachers learning an the acquisition of professional knowledge: An examinations of research on contemporary professional development. In. A. Iran-Nejad, P. Pearson. Review of Research in Education (pp. 173-209). Washington, American Educational Research Association.

Withford, B. \& Jones, K. (1998). Assessment and Accountability in Kentucky: How High Stakes Affects Teaching and Learning. Hargreaves, A. (1998). (Ed.) International Handbook of Educational Change (1163-1178). Gran Bretaña: Kluwer.

Wood, D. (1996). Teacher Cognition in Language Teaching. Beliefs, decision-making and classroom practice. Cambridge: Cambridge University Press.

Young, K. \& Kline, T. (1996). Perceived self-efficacy, outcome-efficacy and feedback: Their effects on professor' teaching development motivation. Canadien Journal of Behavioral Science, 28.

Yus, A. (1999). La evaluación externa como mecanismo de desarrollo profesional. Serie de Monografias, $\mathrm{N}^{\circ} 32$, pp. 45-56. 
